\title{
The PYRIN domain: a novel motif found in apoptosis and inflammation proteins
}

\begin{abstract}
Dear Editor,
Modular protein-protein interaction domains play an important role in many intracellular signal transduction pathways. ${ }^{1}$ In apoptosis signaling pathways, three major families of protein modules have been identified thus far: the death domain, the death effector domain (DED) and the caspase recruitment domain (CARD). ${ }^{2}$ These protein modules of approximately 100 amino acids in length function to mediate homotypic, protein-protein interactions between signaling components leading to the activation of specific downstream targets by stress and developmental stimuli. The interchange of protein modules between signaling molecules has allowed nature to rapidly evolve new signal transduction pathways with expanded combinations of protein interactions. We propose here that the PYRIN domain is a novel protein module that likely mediates protein-protein interactions in apoptotic and inflammatory signaling pathways.
\end{abstract}

CARD4 (also called NOD1) is a CED-4/Apaf-1 family member that activates NF- $\kappa$ B signaling and induces apoptosis. ${ }^{3-5}$ CARD4 contains three putative functional domains: an N-terminal CARD that binds to and activates RICK; a central nucleotide-binding site (NBS) domain involved in protein activation and oligomerization; and a C-terminal domain consisting of leucine-rich repeats that likely functions as a site for interactions with upstream signaling components (LRR; Figure 1A). The structure of CARD4 is similar to the NBS/LRR family of plant proteins that induce localized cell death and changes in gene expression in response to pathogen infection. ${ }^{6}$ However, plant NBS/LRR family members utilize either a leucine zipper motif or a Toll/interleukin-1 receptor homology domain in place of a CARD domain to mediate downstream interactions (Figure 1A). We therefore reasoned that mammalian NBS/LRR family members might use protein modules other than CARD domains as their $\mathrm{N}$-terminal effector domains. To identify such protein modules, we performed a BLASTP search of the protein database using the NBS domain of CARD4 (residues 199-398) and identified two mammalian NBS/LRR proteins of unknown function and structure (accession numbers AK000517 and AB023143). These proteins were named NBS1 (for nucleotide-binding site protein 1) and CARD7 (for CARD protein 7), respectively (Figure 1A). Interestingly, a CARD domain was found at the C-terminus of CARD7 indicating a role for this protein in apoptotic signaling through interactions with other CARD family members. To identify domains at the N-terminal regions of NBS1 and CARD7, we performed a BLASTP search of the public protein database using residues $1-100$ of each protein. Although known protein modules were absent, the $\mathrm{N}$-terminus of both NBS1 and CARD7 (residues 1-100) displayed a strong similarity to the highly conserved $\mathrm{N}$-terminal region of human, mouse and rat pyrin (NBS1: 24\% identity, 50\% similarity; CARD7: $25 \%$ identity, $50 \%$ similarity, Figure $1 \mathrm{~A}, \mathrm{~B}){ }^{7}$ Mutations within the human gene encoding pyrin cause familial Mediterranean fever, an inherited disorder that is characterized by episodes of fever and serosal inflammation. ${ }^{8}$ Although the function of pyrin remains to be determined, it has been proposed to regulate inflammatory signaling in myeloid cells. We therefore named this region of similarity between NBS1, CARD7 and pyrin proteins, the PYRIN domain. Since the N-terminal regions of NBS/LRR family members function as protein interacting modules, we can speculate that the PYRIN domain of NBS1, CARD7 and pyrin mediate interactions with other proteins. Further analysis revealed that the PYRIN domain is also present within the prodomain of zebrafish caspase-13 and in the Nterminal region of the apoptotic protein ASC (Figure $1 A, B) .{ }^{9,10}$ Since caspase prodomains function as proteinprotein interaction domains involved in oligomerization and activation, it is likely that the PYRIN domain of zebrafish caspase-13 mediates binding to other proteins. Interestingly, ASC has a bipartite domain structure consisting of an $\mathrm{N}$-terminal PYRIN domain and a C-terminal CARD domain (Figure 1A). Thus, ASC may function as a FADD-like adaptor protein that binds multiple proteins via its $\mathrm{N}$ - and $\mathrm{C}$ terminal domains.

We have identified the PYRIN domain as a novel protein module found in proteins that are thought to function in apoptotic and inflammatory signaling pathways. It will be of interest to determine whether this domain mediates PYRIN/ PYRIN homotypic interactions or alternatively, heterotypic interactions with other apoptosis protein modules (e.g., death, DED, and/or CARD domains). Further analysis of the PYRIN domain-containing proteins should provide further insight into apoptosis and inflammation signaling pathways.

\section{J Bertin ${ }^{*}, 1$ and PS DiStefano ${ }^{1}$}

\footnotetext{
1 Millennium Pharmaceuticals Inc., Cambridge, MA 02139, USA

* Corresponding author: J Bertin, Millennium Pharmaceuticals Inc., 640 Memorial Drive, Cambridge, MA 02139, USA. Tel: 617-679-7215; Fax: 617679-7071; E-mail: bertin@mpi.com
} 


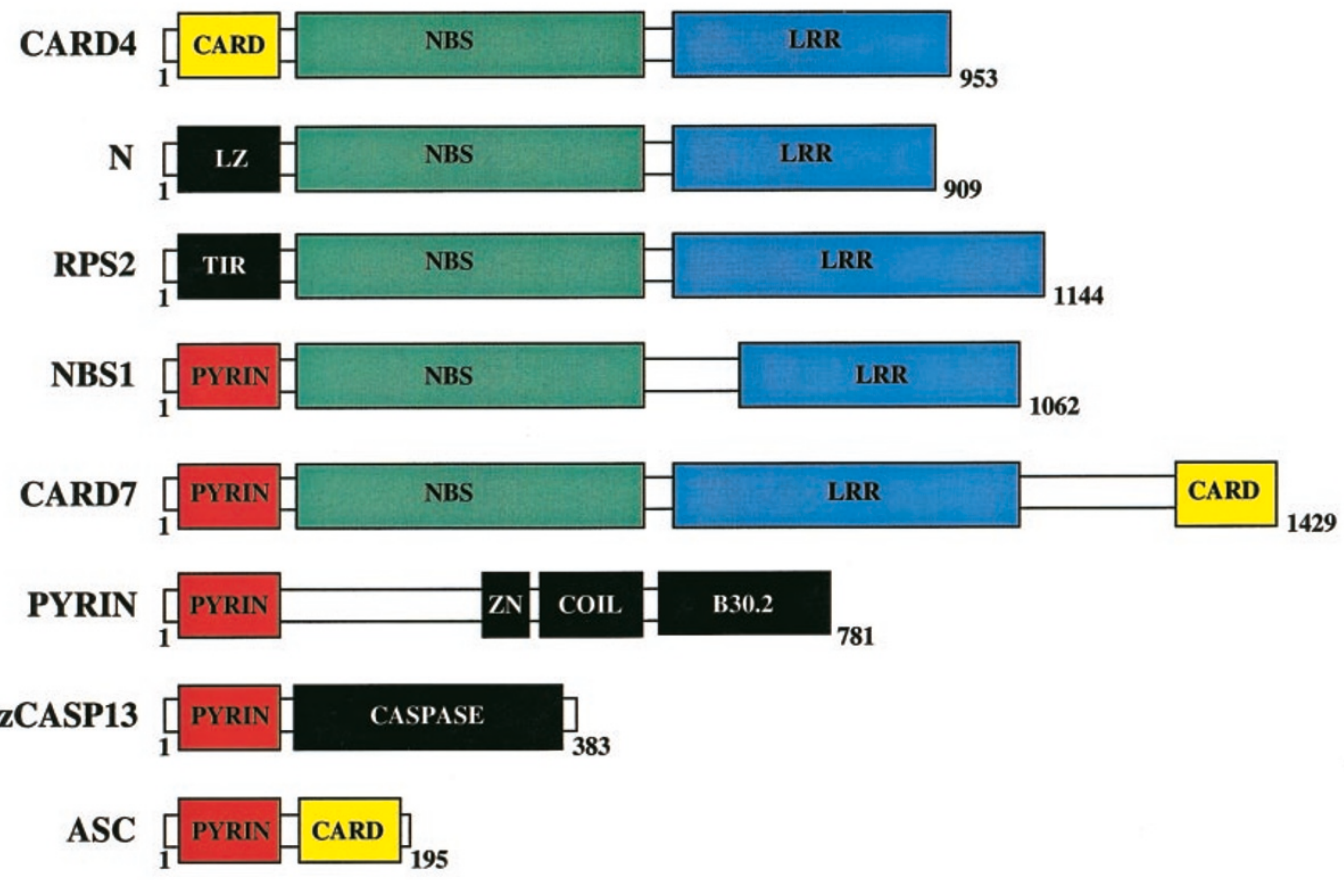

$\mathbf{B}$

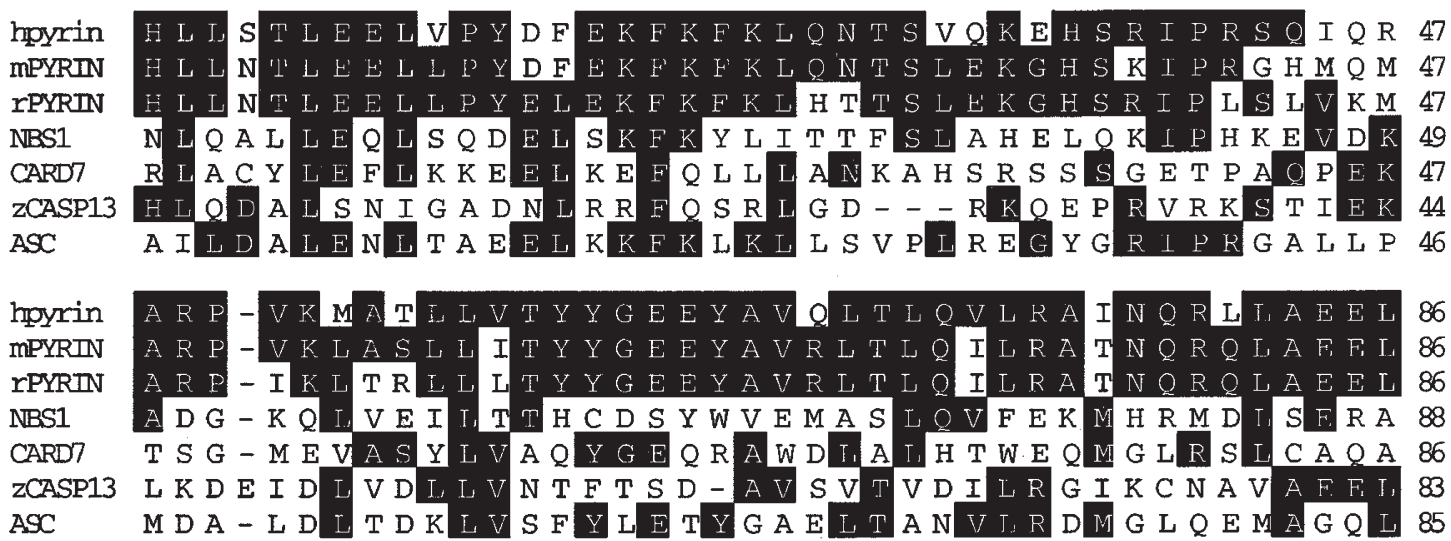

Figure 1 (A) Domain structure of NBS/LRR and PYRIN domain-containing proteins: CARD4, N (Tobacco, ${ }^{11}$ RPS2 (Arabidopsis), ${ }^{12}$ NBS1, CARD7, Pyrin (human), zCASP13 (zebrafish caspase-13) and ASC. CARD (CARD domain, yellow boxes); LZ (leucine zipper domain, black box); TIR (toll/interleukin-1 receptor homology domain, black box); PYRIN (PYRIN domain, red boxes); NBS (nucleotide-binding site, green boxes); LRR (leucine-rich repeats, blue boxes); Zn (B-box zinc finger domain, black box); Coil (Coiled-coil domain, black box); B30.2 domain (black box) and CASPASE (large and small caspase enzymatic subunits, black box). (B) Alignment of PYRIN domains. Black shading indicates identical residues. Human pyrin (AF111163); mouse pyrin (AF143409); rat pyrin (AF143410); NBS1 (AF298547); CARD7 (AF298548); zebrafish caspase-13 (AF233434) and ASC (AB023416)

\footnotetext{
1. Pawson T and Nash P (2000) Genes Dev. 14: 1027-1047

2. Hofmann K (1999) Cell. Mol. Life Sci. 55: 1113-1128

3. Bertin J et al. (1999) J. Biol. Chem. 274: 12955-12958

4. Inohara N et al. (1999) J. Biol. Chem. 274, 14560-14567

5. Inohara N et al. (2000) J. Biol. Chem. 275, 27823-27831

6. Baker B et al. (1997) Science 276: 726-733
}

\author{
7. Chae J et al. (2000) Mamm. Genome 11: 428-435 \\ 8. The International FMF Consortium 1997) 90: 797-807 \\ 9. Inohara N and Nunez G (2000) Cell Death Differ. 7: $509-510$ \\ 10. Masumoto J et al. (1999) J. Biol. Chem. 274: 33835-33838 \\ 11. Whitham S et al. (1994) Cell 78: 1101-1115 \\ 12. Mindrinos M et al. (1994) Cell 78: 1089-1099
}

